International Journal of Modern Physics B

(C) World Scientific Publishing Company

\title{
Classification and Quantification of Entangled Bipartite Qutrit Pure States
}

\author{
Feng Pan*, Guoying Lu \\ Department of Physics, Liaoning Normal University, Dalian 116029, P. R. China \\ *daipan@dlut.edu.cn \\ J. P. Draayer \\ Department of Physics and Astronomy, Louisiana State University, Baton Rouge, LA \\ 70803-4001, USA \\ draayer@sura.org
}

Received Day Month Year

Revised Day Month Year

\begin{abstract}
A complete analysis of entangled bipartite qutrit pure states is carried out based on a simple entanglement measure. An analysis of all possible extremally entangled pure bipartite qutrit states is shown to reduce, with the help of SLOCC transformations, to three distinct types. The analysis and the results should be helpful for finding different entanglement types in multipartite pure state systems.
\end{abstract}

Keywords: Bipartite qutrit pure states; entanglement measure; extremal entanglement; SLOCC.

Quantum entanglement is an important concept in quantum information processing and quantum communication protocols. ${ }^{1}$ Interest in multi-dimensional entangled states comes from the foundations of quantum mechanics as well as the development of new protocols in quantum communication. For example, it has been shown that maximally entangled states of two quantum systems in a highdimensional Hilbert space, qudits, violate local realism stronger than qubits, and entangled qudits are less affected by noise than entangled qubits. ${ }^{2,3}$ In quantum cryptography, ${ }^{4}$ the use of entangled qutrits ${ }^{4,5}$ or qudits ${ }^{6,7}$ instead of qubits is more secure against eavesdropping attacks. Furthermore, the protocols for quantum teleportation or for quantum cryptography work best with maximally entangled states. These facts motivate the development of techniques to generate entangled states among quantum systems in a higher dimensional Hilbert space with good entanglement characteristics. Technical developments in this direction have been made. For example, four polarized entangled photons have been used to form two entangled qutrits. $^{8}$ Entangled qutrits with two photons using an unbalanced 3-arm fiber optic interferometer or photonic orbital angular momentum have been demonstrated. ${ }^{9,10}$ Time-bin entangled qudits of up to 11 dimensions from pump pulses generated by a mode-locked laser have also been reported. ${ }^{11}$ In short, quantifying the entanglement 
measure of a qutrit system is of physical interest.

In this paper, a complete analysis of entangled bipartite qutrit pure states will be carried out based on a simple entanglement measure ${ }^{12}$ and a recent study of the classification of triqubit entangled states. ${ }^{13}$ An analysis of all possible extremally entangled bipartite qutrit pure states with up to six terms is shown to reduce, with the help of stocastic local operation and classical communication (SLOCC) transformations, to three distinct types. The analysis presented here, together with other previously reported results, should be helpful in finding different entanglement types in other multipartite qudit pure state systems.

Based on the method shown in Refs. 13 and 14, only entanglement properties at the single copy level will be considered; asymptotic properties will not be discussed. In such cases, it is well known that two pure states can always be transformed with certainty from each other by means of a LOCC if and only if they are related by a Local Unitary transformation (LU). However, even in bipartite cases, entangled states are not always related by a $\mathrm{LU}$, and continuous parameters are needed to label all equivalence classes. Hence, it seems that one needs to deal with infinitely many kinds of entanglement. Fortunately, such arbitrariness has been overcome with the help of SLOCC. ${ }^{15}$ As defined in Ref. 15, states $\Psi$ and $\Phi$ are equivalent under SLOCC if an Invertible Local Operator (ILO) relating the two states exists, which is denoted as $Q_{A}$ and $Q_{B}$ for the ILOs for particles $A$ and $B$, respectively. Typically, these ILOs are elements of the complex general linear group $G L_{A}(2, c) \otimes G L_{B}(2, c)$ where each copy operates on the corresponding local basis.

According to the definition, ${ }^{12}$ for a $N$-particle qutrit pure state $\psi$, the entanglement measure can be defined as

$$
\eta(\Psi)=\left\{\begin{array}{ccc}
\frac{1}{N} \sum_{i=1}^{N} S_{i} & \text { if } \quad S_{i} \neq 0 \forall i \\
0 & \text { if } \quad S_{i}=0,
\end{array}\right.
$$

where $S_{i}=-\operatorname{Tr}\left[\left(\rho_{\Psi}\right)_{i} \log _{3}\left(\rho_{\Psi}\right)_{i}\right]$ is the reduced von Neumann entropy for the ith particle only, with the other $N-1$ particles traced out, and $\left(\rho_{\Psi}\right)_{i}$ is the corresponding reduced density matrix. In the case of $N=2,(1)$ reduces to the well-known entanglement measure for bipartite pure states, in which we use the logarithm to the base 3 instead of base 2 to ensure that the maximal measure is normalized to 1 in the qutrit case. It can be verified that the state $\Psi$ is partially separable when one of the reduced von Neumann entropies $S_{i}$ is zero. In such cases the state $\Psi$ is not a genuine entangled N-qutrit state. Furthermore, definition (1) is invariant under $\mathrm{LU}$, which is equivalent to LOCC for a pure state system. ${ }^{12,13}$

Instead of qubit state, single-particle qutrit states can be denoted by $|1\rangle,|0\rangle$, and $|-1\rangle$, which are assumed to be mutually orthonormal. Product states of a bipartite qutrit system are denoted by 


$$
\begin{aligned}
& \left\{\left|U_{1}\right\rangle=|11\rangle, \quad\left|U_{2}\right\rangle=|10\rangle, \quad\left|U_{3}\right\rangle=|1-1\rangle,\right. \\
& \left|V_{1}\right\rangle=|01\rangle, \quad\left|V_{2}\right\rangle=|00\rangle, \quad\left|V_{3}\right\rangle=|0-1\rangle, \\
& \left.\left|W_{1}\right\rangle=|-11\rangle, \quad\left|W_{2}\right\rangle=|-10\rangle, \quad\left|W_{3}\right\rangle=|-1-1\rangle\right\} .
\end{aligned}
$$

These configurations span a 9-dimensional Hilbert subspace and any bipartite qutrit pure state can be expanded in terms of them.

For two-term cases, when a state is a linear combination of a pair of states among $\left\{\left(\left|U_{i}\right\rangle,\left|U_{j}\right\rangle\right) ;\left(\left|V_{i}\right\rangle,\left|V_{j}\right\rangle\right) ;\left(\left|W_{i}\right\rangle,\left|W_{j}\right\rangle\right)\right\}$, or $\left(\left|U_{i}\right\rangle,\left|V_{i}\right\rangle\right) ;\left(\left|U_{i}\right\rangle,\left|W_{i}\right\rangle\right)$; $\left(\left|V_{i}\right\rangle,\left|W_{i}\right\rangle\right)$, where $i, j \in P, i \neq j, P=\{1,2,3\}$, it can always be decomposed into a bipartite product state. Such states are separable and disentangled. There are 18 such combinations. When a state is a linear combination of a pair of states among $\left(\left|U_{i}\right\rangle,\left|V_{j}\right\rangle\right) ;\left(\left|U_{i}\right\rangle,\left|W_{j}\right\rangle\right)$; or $\left(\left|V_{i}\right\rangle,\left|W_{j}\right\rangle\right)$, it is a genuinely entangled state, where $i, j \in P, i \neq j, P=\{1,2,3\}$. There are also 18 such linear combinations in total. Take $|\Psi\rangle=a\left|U_{1}\right\rangle+b e^{i \alpha}\left|V_{2}\right\rangle=a|11\rangle+b e^{i \alpha}|00\rangle$ as an example, where $a, b \neq 0$ are real and satisfy the normalization condition $a^{2}+b^{2}=1$, and $\alpha$ is a relative phase. Then, to maximize its measure (1) with the normalization condition, one can find parameters for the corresponding extremal cases. It can be verified easily that $\eta=0.63093$ when $a=b=\frac{1}{\sqrt{2}}$ in such cases, and there is no restriction on relative phase. We call such configurations Type I entangled bipartite qutrit states.

For three term cases, if a state is a linear combination of $\left(\left|U_{i}\right\rangle,\left|U_{j}\right\rangle,\left|U_{k}\right\rangle\right)$; $\left(\left|V_{i}\right\rangle,\left|V_{j}\right\rangle,\left|V_{k}\right\rangle\right) ;\left(\left|W_{i}\right\rangle,\left|W_{j}\right\rangle,\left|W_{k}\right\rangle\right)$, where $i, j, k \in P$ and $i \neq j \neq k$, or $\left(\left|U_{i}\right\rangle,\left|V_{i}\right\rangle,\left|W_{i}\right\rangle\right)$, where $i \in P, P=\{1,2,3\}$, it can always be decomposed into a bipartite product state. Therefore, these combinations are also separable. When a state is $a\left|U_{i}\right\rangle+b e^{i \alpha}\left|V_{j}\right\rangle+c e^{i \beta}\left|W_{k}\right\rangle$, where $a, b, c \neq 0$ are real and satisfy the normalization condition $a^{2}+b^{2}+c^{2}=1$, and $\alpha, \beta$ are relative phases, it is called a Type $\mathrm{III}_{1}$ state. There are 6 such equivalent linear combinations. To maximize its measure (1) with the constraint $a^{2}+b^{2}+c^{2}=1$, one can find parameters for the corresponding extremal cases. It can be verified easily that $\eta_{\max }=1$ when $a=b=c=\frac{1}{\sqrt{3}}$ for such cases, where there is no restriction on relative phase. It can be proven that the Type $\mathrm{III}_{1}$ states are inequivalent to the Type I states. To do so, we choose one representative of Type $\mathrm{III}_{1}$ states,

$$
|\Psi\rangle=x_{1}|11\rangle+x_{2}|00\rangle+x_{3}|-1-1\rangle=\left(x_{1}|11\rangle+x_{2}|00\rangle\right)+x_{3}|-1-1\rangle
$$

with nonzero $x_{1}, x_{2}$, and $x_{3}$. It is clear that the first two terms in (4) form a Type I state, which remains Type I under the SLOCC, while the last term is always a bipartite product state. Since all coefficients are nonzero in the case considered, the state in (4) remains a linear combination of Type I entangled state and a bipartite product state. It will degenerate into a Type I state only when $x_{3} \rightarrow 0$. Thus, we have proven that Type $\mathrm{III}_{1}$ is inequivalent to a Type I state, which will be called Type II bipartite entangled qutrit states.

If a state is a linear combination of $\left(\left|U_{i}\right\rangle,\left|U_{j}\right\rangle,\left|V_{k}\right\rangle\right.$ or $\left.\left|W_{k}\right\rangle\right) ;\left(\left|V_{i}\right\rangle,\left|V_{j}\right\rangle,\left|U_{k}\right\rangle\right.$ or $\left.\left|W_{k}\right\rangle\right)$; $\left(\left|W_{i}\right\rangle,\left|W_{j}\right\rangle,\left|U_{k}\right\rangle\right.$ or $\left.\left|V_{k}\right\rangle\right)$, where $i, j \in P, k=i$ or $k=j$ and $i \neq j$, 
$P=\{1,2,3\}$, it is called a Type $\mathrm{III}_{2}$ state. There are a total of 36 such combinations. In such cases, no extremal value of $\eta$ with all coefficients non zero exists. When a state is a linear combination of $\left\{\left(\left|U_{i}\right\rangle,\left|U_{j}\right\rangle,\left|V_{k}\right\rangle\right.\right.$ or $\left.\left|W_{k}\right\rangle\right),\left(\left|V_{i}\right\rangle,\left|V_{j}\right\rangle,\left|U_{k}\right\rangle\right.$ or $\left.\left|W_{k}\right\rangle\right),\left(\left|W_{i}\right\rangle,\left|W_{j}\right\rangle,\left|U_{k}\right\rangle\right.$ or $\left.\left.\left|V_{k}\right\rangle\right)\right\}$, or $\left\{\left(\left|U_{i}\right\rangle,\left|V_{i}\right\rangle,\left|W_{j}\right\rangle\right),\left(\left|U_{i}\right\rangle,\left|W_{i}\right\rangle,\left|V_{j}\right\rangle\right)\right\}$, $\left.\left(\left|V_{i}\right\rangle,\left|W_{i}\right\rangle,\left|U_{j}\right\rangle\right)\right\}$, where $i, j, k \in P, i \neq j \neq k, P=\{1,2,3\}$, it is called a Type $\mathrm{III}_{3}$ state. There are 36 such combinations. Take $|\Psi\rangle=a\left|U_{1}\right\rangle+b e^{i \alpha}\left|U_{2}\right\rangle+c e^{i \beta}\left|V_{3}\right\rangle=$ $a|11\rangle+b e^{i \alpha}|10\rangle+c e^{i \beta}|0-1\rangle$ as an example, where $a, b, c \neq 0$ are real and satisfy the normalization condition $a^{2}+b^{2}+c^{2}=1$, and $\alpha, \beta$ are relative phases. It can be shown with conditional maximization that the extremal value of $\eta=0.63093$ in this case when $a^{2}+b^{2}=\frac{1}{2}$ and $c=\frac{1}{2}$, which is independent of the relative phases. Using SLOCC, one can prove that Type $\mathrm{III}_{3}$ states are equivalent to the Type I states.

For four term cases, there are $\left(\begin{array}{l}9 \\ 4\end{array}\right)=126$ linear combinations chosen from (3) that cannot be reduced into bipartite product states. All these states are classified into 5 types and listed in Table 1, for which there is no extremal value of $\eta$ for the Type $\mathrm{IV}_{1}, \mathrm{IV}_{2}$, and $\mathrm{IV}_{3}$ cases with all coefficients nonzero. Actually, these states degenerate into Type I states under SLOCC. For the Type $\mathrm{IV}_{4}$ case, we take $|\Psi\rangle=a\left|U_{1}\right\rangle+b e^{i \alpha}\left|U_{2}\right\rangle+c e^{i \beta}\left|V_{1}\right\rangle+d e^{i \gamma}\left|V_{2}\right\rangle$ as an example, where $a, b, c, d \neq 0$ are real and satisfy the normalization condition $a^{2}+b^{2}+c^{2}+d^{2}=1$, and $\alpha, \beta, \gamma$ are relative phases. One can verify that $\eta=0.63093$ is its extremal value when $a=d, b=c$, and $\varphi=\alpha+\beta-\gamma=2 k \pi+\pi$. Therefore, such states are also equivalent to Type I entangled states under SLOCC. For the Type $\mathrm{IV}_{5}$ case, we take $|\Psi\rangle=a\left|U_{1}\right\rangle+b e^{i \alpha}\left|U_{2}\right\rangle+c e^{i \beta}\left|V_{3}\right\rangle+d e^{i \gamma}\left|W_{3}\right\rangle$ as an example, where $a, b, c, d \neq 0$ are real and satisfy the normalization condition $a^{2}+b^{2}+c^{2}+d^{2}=1$, and $\alpha, \beta, \gamma$ are relative phases. It can be shown that there is an extremal value with $\eta=0.63093$ when $a^{2}+b^{2}=\frac{1}{2}$ and $c^{2}+d^{2}=\frac{1}{2}$, which is independent of the relative phases. It can also be proven that these Type $\mathrm{IV}_{5}$ states can be transformed into Type I states under SLOCC. Thus, four-term bipartite qutrit states are all equivalent to the Type I states; no new type of entangled states are found in this case.

For five term cases, there are also a total of $\left(\begin{array}{l}9 \\ 5\end{array}\right)=126$ linear combinations, of which all are genuine entangled states. They are classified into 6 types as shown in Table 2. For the Type $\mathrm{V}_{1}$ case, we take $|\Psi\rangle=a\left|U_{1}\right\rangle+b e^{i \alpha}\left|U_{2}\right\rangle+c e^{i \beta}\left|U_{3}\right\rangle+d e^{i \gamma}\left|V_{1}\right\rangle+$ $f e^{i \xi}\left|V_{2}\right\rangle$ as an example, where $a, b, c, d, f \neq 0$ are real and satisfy normalization condition $a^{2}+b^{2}+c^{2}+d^{2}+f^{2}=1$, and $\alpha, \beta, \gamma, \xi$ are relative phase factors. It can be verified that $\eta=0.63093$ when $a^{2}+b^{2}+c^{2}=\frac{1}{2}, d^{2}+f^{2}=\frac{1}{2}$, and $\varphi=\alpha+\gamma-\xi=$ $2 k \pi+\pi$. Such a state is equivalent to a Type I entangled state under SLOCC. For the Type $\mathrm{V}_{2}$ case, we take $|\Psi\rangle=a\left|U_{1}\right\rangle+b e^{i \alpha}\left|U_{2}\right\rangle+c e^{i \beta}\left|V_{1}\right\rangle+d e^{i \gamma}\left|V_{2}\right\rangle+f e^{i \xi}\left|W_{3}\right\rangle$ as an example, where $a, b, c, d, f \neq 0$ are real and satisfy the normalization condition $a^{2}+b^{2}+c^{2}+d^{2}+f^{2}=1$, and $\alpha, \beta, \gamma, \xi$ are relative phases. It can be proven that there is an extremal value $\eta=0.63093$ when $a=b=c=d=\frac{1}{2 \sqrt{2}}, f=\frac{1}{\sqrt{2}}$, and $\varphi=\alpha+\beta-\gamma=2 k \pi$. One can prove that Type $\mathrm{V}_{2}$ states are equivalent to Type I entangled states under SLOCC. In addition, there is another extremal value $\eta=1$ 
Table 1. Classification of entangled states with four terms

\begin{tabular}{|c|c|c|c|}
\hline Types & & Linear Combinations & \\
\hline \multirow[t]{4}{*}{$\mathrm{IV}_{1}$} & $\left(\left|U_{i}\right\rangle,\left|U_{j}\right\rangle,\left|U_{k}\right\rangle,\left|V_{m}\right\rangle\right)$ & $\left(\left|U_{i}\right\rangle,\left|U_{j}\right\rangle,\left|U_{k}\right\rangle,\left|W_{m}\right\rangle\right)$ & $\left(\left|V_{i}\right\rangle,\left|V_{j}\right\rangle,\left|V_{k}\right\rangle,\left|U_{m}\right\rangle\right)$ \\
\hline & $\begin{array}{l}\left(\left|V_{i}\right\rangle,\left|V_{j}\right\rangle,\left|V_{k}\right\rangle,\left|W_{m}\right\rangle\right) \\
\quad i, j, k, m \in P\end{array}$ & $\begin{array}{c}\left(\left|W_{i}\right\rangle,\left|W_{j}\right\rangle,\left|W_{k}\right\rangle,\left|U_{m}\right\rangle\right) \\
P=\{1,2,3\}\end{array}$ & $\begin{array}{c}\left(\left|W_{i}\right\rangle,\left|W_{j}\right\rangle,\left|W_{k}\right\rangle,\left|V_{m}\right\rangle\right) \\
i \neq j \neq k .\end{array}$ \\
\hline & $\left(\left|U_{i}\right\rangle,\left|U_{j}\right\rangle,\left|V_{i}\right\rangle,\left|W_{i}\right\rangle\right)$ & $\left(\left|U_{i}\right\rangle,\left|U_{j}\right\rangle,\left|V_{j}\right\rangle,\left|W_{j}\right\rangle\right)$ & $\left(\left|V_{i}\right\rangle,\left|V_{j}\right\rangle,\left|U_{i}\right\rangle,\left|W_{i}\right\rangle\right)$ \\
\hline & $\begin{array}{c}\left(\left|V_{i}\right\rangle,\left|V_{j}\right\rangle,\left|U_{j}\right\rangle,\left|W_{j}\right\rangle\right) \\
i, j \in P\end{array}$ & $\begin{array}{c}\left(\left|W_{i}\right\rangle,\left|W_{j}\right\rangle,\left|U_{i}\right\rangle,\left|V_{i}\right\rangle\right) \\
P=\{1,2,3\}\end{array}$ & $\begin{array}{c}\left(\left|W_{i}\right\rangle,\left|W_{j}\right\rangle,\left|U_{j}\right\rangle,\left|V_{j}\right\rangle\right) \\
i \neq j .\end{array}$ \\
\hline \multirow[t]{4}{*}{$\mathrm{IV}_{2}$} & $\left(\left|U_{i}\right\rangle,\left|U_{j}\right\rangle,\left|V_{i}\right\rangle,\left|V_{k}\right\rangle\right)$ & $\left(\left|U_{i}\right\rangle,\left|U_{j}\right\rangle,\left|V_{j}\right\rangle,\left|V_{k}\right\rangle\right)$ & $\left(\left|U_{i}\right\rangle,\left|U_{j}\right\rangle,\left|W_{i}\right\rangle,\left|W_{k}\right\rangle\right)$ \\
\hline & $\begin{array}{c}\left(\left|U_{i}\right\rangle,\left|U_{j}\right\rangle,\left|W_{j}\right\rangle,\left|W_{k}\right\rangle\right) \\
i, j, k \in P\end{array}$ & $\begin{array}{c}\left(\left|V_{i}\right\rangle,\left|V_{j}\right\rangle,\left|W_{i}\right\rangle,\left|W_{k}\right\rangle\right) \\
P=\{1,2,3\}\end{array}$ & $\begin{array}{c}\left(\left|V_{i}\right\rangle,\left|V_{j}\right\rangle,\left|W_{j}\right\rangle,\left|W_{k}\right\rangle\right) \\
i \neq j \neq k .\end{array}$ \\
\hline & $\left(\left|U_{i}\right\rangle,\left|U_{j}\right\rangle,\left|V_{i}\right\rangle,\left|W_{j}\right\rangle\right)$ & $\left(\left|U_{i}\right\rangle,\left|U_{j}\right\rangle,\left|V_{j}\right\rangle,\left|W_{i}\right\rangle\right)$ & $\left(\left|V_{i}\right\rangle,\left|V_{j}\right\rangle,\left|U_{i}\right\rangle,\left|W_{j}\right\rangle\right)$ \\
\hline & $\begin{array}{c}\left(\left|V_{i}\right\rangle,\left|V_{j}\right\rangle,\left|U_{j}\right\rangle,\left|W_{i}\right\rangle\right) \\
i, j \in P\end{array}$ & $\begin{array}{c}\left(\left|W_{i}\right\rangle,\left|W_{j}\right\rangle,\left|U_{i}\right\rangle,\left|V_{j}\right\rangle\right) \\
P=\{1,2,3\}\end{array}$ & $\begin{array}{c}\left(\left|W_{i}\right\rangle,\left|W_{j}\right\rangle,\left|U_{j}\right\rangle,\left|V_{i}\right\rangle\right) \\
i \neq j .\end{array}$ \\
\hline \multirow[t]{4}{*}{$\mathrm{IV}_{3}$} & $\left(\left|U_{i}\right\rangle,\left|U_{j}\right\rangle,\left|V_{i}\right\rangle,\left|W_{k}\right\rangle\right)$ & $\left(\left|U_{i}\right\rangle,\left|U_{j}\right\rangle,\left|V_{j}\right\rangle,\left|W_{k}\right\rangle\right)$ & $\left(\left|U_{i}\right\rangle,\left|U_{j}\right\rangle,\left|W_{i}\right\rangle,\left|V_{k}\right\rangle\right)$ \\
\hline & $\left(\left|U_{i}\right\rangle,\left|U_{j}\right\rangle,\left|W_{j}\right\rangle,\left|V_{k}\right\rangle\right)$ & $\left(\left|V_{i}\right\rangle,\left|V_{j}\right\rangle,\left|U_{i}\right\rangle,\left|W_{k}\right\rangle\right)$ & $\left(\left|V_{i}\right\rangle,\left|V_{j}\right\rangle,\left|U_{j}\right\rangle,\left|W_{k}\right\rangle\right)$ \\
\hline & $\left(\left|V_{i}\right\rangle,\left|V_{j}\right\rangle,\left|W_{i}\right\rangle,\left|U_{k}\right\rangle\right)$ & $\left(\left|V_{i}\right\rangle,\left|V_{j}\right\rangle,\left|W_{j}\right\rangle,\left|U_{k}\right\rangle\right)$ & $\left(\left|W_{i}\right\rangle,\left|W_{j}\right\rangle,\left|U_{i}\right\rangle,\left|V_{k}\right\rangle\right)$ \\
\hline & $\begin{array}{c}\left(\left|W_{i}\right\rangle,\left|W_{j}\right\rangle,\left|U_{j}\right\rangle,\left|V_{k}\right\rangle\right) \\
i, j \in P\end{array}$ & $\begin{array}{c}\left(\left|W_{i}\right\rangle,\left|W_{j}\right\rangle,\left|U_{k}\right\rangle,\left|V_{i}\right\rangle\right) \\
P=\{1,2,3\}\end{array}$ & $\begin{array}{c}\left(\left|W_{i}\right\rangle,\left|W_{j}\right\rangle,\left|U_{k}\right\rangle,\left|V_{j}\right\rangle\right) \\
i \neq j \neq k .\end{array}$ \\
\hline $\mathrm{IV}_{4}$ & $\begin{array}{c}\left(\left|U_{i}\right\rangle,\left|U_{j}\right\rangle,\left|V_{i}\right\rangle,\left|V_{j}\right\rangle\right) \\
i, j \in P\end{array}$ & $\begin{array}{c}\left(\left|U_{i}\right\rangle,\left|U_{j}\right\rangle,\left|W_{i}\right\rangle,\left|W_{j}\right\rangle\right) \\
P=\{1,2,3\}\end{array}$ & $\begin{array}{c}\left(\left|V_{i}\right\rangle,\left|V_{j}\right\rangle,\left|W_{i}\right\rangle,\left|W_{j}\right\rangle\right) \\
i \neq j\end{array}$ \\
\hline $\mathrm{IV}_{5}$ & $\begin{array}{c}\left(\left|U_{i}\right\rangle,\left|U_{j}\right\rangle,\left|V_{k}\right\rangle,\left|W_{k}\right\rangle\right) \\
i, j, k \in P\end{array}$ & $\begin{array}{c}\left(\left|V_{i}\right\rangle,\left|V_{j}\right\rangle,\left|U_{k}\right\rangle,\left|W_{k}\right\rangle\right) \\
P=\{1,2,3\}\end{array}$ & $\begin{array}{c}\left(\left|W_{i}\right\rangle,\left|W_{j}\right\rangle,\left|U_{k}\right\rangle,\left|V_{k}\right\rangle\right) \\
i \neq j \neq k .\end{array}$ \\
\hline
\end{tabular}

when $a=b=c=d=\frac{1}{\sqrt{6}}, f=\frac{1}{\sqrt{3}}$, and $\varphi=\alpha+\beta-\gamma=2 k \pi+\pi$, of which the corresponding states are equivalent to Type II entangled states under SLOCC. Furthermore, there is no extremal value found for the Type $\mathrm{V}_{3-6}$ states with all coefficients nonzero. Therefore, there is no new type of entangled states with linear combination of five terms; they can all be transformed either into Type I or Type 
II states under SLOCC.

For six term cases, there are $\left(\begin{array}{l}9 \\ 6\end{array}\right)=84$ linear combinations, all of which are genuine entangled states. They are classified into 4 types as listed in Table 3.

For the Type $\mathrm{VI}_{1}$ case, we take $|\Psi\rangle=a\left|U_{1}\right\rangle+b e^{i \alpha}\left|U_{2}\right\rangle+c e^{i \beta}\left|U_{3}\right\rangle+d e^{i \gamma}\left|V_{1}\right\rangle+$ $f e^{i \xi}\left|V_{2}\right\rangle+g e^{i \sigma}\left|V_{3}\right\rangle$ as an example, where $a, b, c, d, f, g \neq 0$ are real and satisfy the normalization condition $a^{2}+b^{2}+c^{2}+d^{2}+f^{2}+g^{2}=1$, and $\alpha, \beta, \gamma, \xi, \sigma$ are relative phases. It can be shown that there is an extremal value with $\eta=0.63093$ when $a=d, b=f, c=g, \omega=\beta+\gamma-\sigma=2 k \pi+\pi, \varphi=\alpha+\gamma-\xi=2 k \pi$; or when $a=g, b=f, c=d, \omega=\beta+\gamma-\sigma=2 k \pi, \varphi=\alpha+\gamma-\xi=2 k \pi+\pi$, which equals to the extremal value of the entanglement of Type I states. Furthermore, it can be proven that Type $\mathrm{VI}_{1}$ states are equivalent to Type I states under SLOCC. For the Type $\mathrm{VI}_{2}$ case, we take $|\Psi\rangle=a\left|U_{1}\right\rangle+b e^{i \alpha}\left|U_{2}\right\rangle+c e^{i \beta}\left|V_{1}\right\rangle+d e^{i \gamma}\left|V_{3}\right\rangle+f e^{i \xi}\left|W_{2}\right\rangle+g e^{i \sigma}\left|W_{3}\right\rangle$ as an example, where $a, b, c, d, f, g \neq 0$ are real and satisfy the normalization condition $a^{2}+b^{2}+c^{2}+d^{2}+f^{2}+g^{2}=1$, and $\alpha, \beta, \gamma, \xi, \sigma$ are relative phases. It can be verified that there is another extremal value of the entanglement with $\eta=0.78969$ when $a=b=c=d=f=g=\frac{1}{\sqrt{6}}$, and $\varphi=\alpha+\beta-\gamma-\xi+\sigma=2 k \pi$. Obviously, the extremal value of $\eta$ in this case differs from those of Type I and II states.

Table 2. Classification of entangled states with five terms

\begin{tabular}{|c|c|c|c|}
\hline Types & & Linear Combinations & \\
\hline \multirow[t]{4}{*}{$\mathrm{v}_{1}$} & $\left(\left|U_{i}\right\rangle,\left|U_{j}\right\rangle,\left|U_{k}\right\rangle,\left|V_{m}\right\rangle,\left|V_{n}\right\rangle\right)$ & $\left(\left|U_{i}\right\rangle,\left|U_{j}\right\rangle,\left|U_{k}\right\rangle,\left|W_{m}\right\rangle,\left|W_{n}\right\rangle\right)$ & $\left(\left|V_{i}\right\rangle,\left|V_{j}\right\rangle,\left|V_{k}\right\rangle,\left|U_{m}\right\rangle,\left|U_{n}\right\rangle\right)$ \\
\hline & $\begin{array}{c}\left(\left|V_{i}\right\rangle,\left|V_{j}\right\rangle,\left|V_{k}\right\rangle,\left|W_{m}\right\rangle,\left|W_{n}\right\rangle\right) \\
i, \quad j, k, \quad m, \quad n \in P\end{array}$ & $\begin{array}{c}\left(\left|W_{i}\right\rangle,\left|W_{j}\right\rangle,\left|W_{k}\right\rangle,\left|U_{m}\right\rangle,\left|U_{n}\right\rangle\right) \\
P=\{1,2,3\}, i \neq j \neq k\end{array}$ & $\begin{array}{c}\left(\left|W_{i}\right\rangle,\left|W_{j}\right\rangle,\left|W_{k}\right\rangle,\left|V_{m}\right\rangle,\left|V_{n}\right\rangle\right) \\
m \neq n .\end{array}$ \\
\hline & $\left(\left|U_{i}\right\rangle,\left|U_{j}\right\rangle,\left|V_{i}\right\rangle,\left|V_{j}\right\rangle,\left|W_{i}\right\rangle\right)$ & $\left(\left|U_{i}\right\rangle,\left|U_{j}\right\rangle,\left|V_{i}\right\rangle,\left|V_{j}\right\rangle,\left|W_{j}\right\rangle\right)$ & $\left(\left|V_{i}\right\rangle,\left|V_{j}\right\rangle,\left|W_{i}\right\rangle,\left|W_{j}\right\rangle,\left|V_{i}\right\rangle\right)$ \\
\hline & $\begin{array}{c}\left(\left|V_{i}\right\rangle,\left|V_{j}\right\rangle,\left|W_{i}\right\rangle,\left|W_{j}\right\rangle,\left|V_{j}\right\rangle\right) \\
i, j \in P\end{array}$ & $\begin{array}{c}\left(\left|V_{i}\right\rangle,\left|V_{j}\right\rangle,\left|W_{i}\right\rangle,\left|W_{j}\right\rangle,\left|U_{i}\right\rangle\right) \\
P=\{1,2,3\}\end{array}$ & $\begin{array}{c}\left(\left|V_{i}\right\rangle,\left|V_{j}\right\rangle,\left|W_{i}\right\rangle,\left|W_{j}\right\rangle,\left|U_{j}\right\rangle\right) \\
i \neq j\end{array}$ \\
\hline $\mathrm{v}_{2}$ & $\begin{array}{c}\left(\left|U_{i}\right\rangle,\left|U_{j}\right\rangle,\left|V_{i}\right\rangle,\left|V_{j}\right\rangle,\left|W_{k}\right\rangle\right) \\
i, j, k \in P\end{array}$ & $\begin{array}{c}\left(\left|V_{i}\right\rangle,\left|V_{j}\right\rangle,\left|W_{i}\right\rangle,\left|W_{j}\right\rangle,\left|V_{k}\right\rangle\right) \\
P=\{1,2,3\}\end{array}$ & $\begin{array}{c}\left(\left|V_{i}\right\rangle,\left|V_{j}\right\rangle,\left|W_{i}\right\rangle,\left|W_{j}\right\rangle,\left|U_{k}\right\rangle\right) \\
i \neq j \neq k\end{array}$ \\
\hline $\mathrm{v}_{3}$ & $\begin{array}{c}\left(\left|U_{i}\right\rangle,\left|U_{j}\right\rangle,\left|U_{k}\right\rangle,\left|V_{m}\right\rangle,\left|W_{m}\right\rangle\right) \\
i, j, k, \quad m \in P\end{array}$ & $\begin{array}{c}\left(\left|V_{i}\right\rangle,\left|V_{j}\right\rangle,\left|V_{k}\right\rangle,\left|U_{m}\right\rangle,\left|W_{m}\right\rangle\right) \\
P=\{1,2,3\}\end{array}$ & $\begin{array}{c}\left(\left|W_{i}\right\rangle,\left|W_{j}\right\rangle,\left|W_{k}\right\rangle,\left|U_{m}\right\rangle,\left|V_{m}\right\rangle\right) \\
i \neq j \neq k\end{array}$ \\
\hline \multirow[t]{3}{*}{$\mathrm{v}_{4}$} & $\begin{array}{c}\left(\left|U_{i}\right\rangle,\left|U_{j}\right\rangle,\left|U_{k}\right\rangle,\left|V_{m}\right\rangle,\left|W_{n}\right\rangle\right) \\
i, j, k, \quad m, n \in P\end{array}$ & $\begin{array}{c}\left(\left|V_{i}\right\rangle,\left|V_{j}\right\rangle,\left|V_{k}\right\rangle,\left|U_{m}\right\rangle,\left|W_{n}\right\rangle\right) \\
P=\{1,2,3\}\end{array}$ & $\begin{array}{c}\left(\left|W_{i}\right\rangle,\left|W_{j}\right\rangle,\left|W_{k}\right\rangle,\left|U_{m}\right\rangle,\left|V_{n}\right\rangle\right) \\
i \neq j \neq k, m \neq n .\end{array}$ \\
\hline & $\left(\left|U_{i}\right\rangle,\left|U_{j}\right\rangle,\left|V_{i}\right\rangle,\left|V_{k}\right\rangle,\left|W_{i}\right\rangle\right)$ & $\left(\left|U_{i}\right\rangle,\left|U_{j}\right\rangle,\left|V_{j}\right\rangle,\left|V_{k}\right\rangle,\left|W_{j}\right\rangle\right)$ & $\left(\left|U_{i}\right\rangle,\left|U_{j}\right\rangle,\left|W_{i}\right\rangle,\left|W_{k}\right\rangle,\left|V_{i}\right\rangle\right)$ \\
\hline & $\begin{array}{c}\left(\left|U_{i}\right\rangle,\left|U_{j}\right\rangle,\left|W_{j}\right\rangle,\left|W_{k}\right\rangle,\left|V_{j}\right\rangle\right) \\
i, j, k \in P\end{array}$ & $\begin{array}{c}\left(\left|V_{i}\right\rangle,\left|V_{j}\right\rangle,\left|W_{i}\right\rangle,\left|W_{k}\right\rangle,\left|U_{i}\right\rangle\right) \\
P=\{1,2,3\}\end{array}$ & $\begin{array}{c}\left(\left|V_{i}\right\rangle,\left|V_{j}\right\rangle,\left|W_{j}\right\rangle,\left|W_{k}\right\rangle,\left|U_{j}\right\rangle\right) \\
i \neq j \neq k\end{array}$ \\
\hline \multirow[t]{2}{*}{$\mathrm{v}_{5}$} & $\left(\left|U_{i}\right\rangle,\left|U_{j}\right\rangle,\left|V_{i}\right\rangle,\left|V_{k}\right\rangle,\left|W_{j}\right\rangle\right)$ & $\left(\left|U_{i}\right\rangle,\left|U_{j}\right\rangle,\left|V_{j}\right\rangle,\left|V_{k}\right\rangle,\left|W_{i}\right\rangle\right)$ & $\left(\left|V_{i}\right\rangle,\left|V_{j}\right\rangle,\left|W_{i}\right\rangle,\left|W_{k}\right\rangle,\left|V_{j}\right\rangle\right)$ \\
\hline & $\begin{array}{c}\left(\left|V_{i}\right\rangle,\left|V_{j}\right\rangle,\left|W_{j}\right\rangle,\left|W_{k}\right\rangle,\left|V_{i}\right\rangle\right) \\
i, j, k \in P\end{array}$ & $\begin{array}{c}\left(\left|V_{i}\right\rangle,\left|V_{j}\right\rangle,\left|W_{i}\right\rangle,\left|W_{k}\right\rangle,\left|U_{j}\right\rangle\right) \\
P=\{1,2,3\}\end{array}$ & $\begin{array}{c}\left(\left|V_{i}\right\rangle,\left|V_{j}\right\rangle,\left|W_{j}\right\rangle,\left|W_{k}\right\rangle,\left|U_{i}\right\rangle\right) \\
i \neq j \neq k\end{array}$ \\
\hline \multirow[t]{2}{*}{$\mathrm{v}_{6}$} & $\left(\left|U_{i}\right\rangle,\left|U_{j}\right\rangle,\left|V_{i}\right\rangle,\left|V_{k}\right\rangle,\left|W_{k}\right\rangle\right)$ & $\left(\left|U_{i}\right\rangle,\left|U_{j}\right\rangle,\left|V_{j}\right\rangle,\left|V_{k}\right\rangle,\left|W_{k}\right\rangle\right)$ & $\left(\left|V_{i}\right\rangle,\left|V_{j}\right\rangle,\left|W_{i}\right\rangle,\left|W_{k}\right\rangle,\left|V_{k}\right\rangle\right)$ \\
\hline & $\begin{array}{c}\left(\left|V_{i}\right\rangle,\left|V_{j}\right\rangle,\left|W_{j}\right\rangle,\left|W_{k}\right\rangle,\left|V_{k}\right\rangle\right) \\
i, j, k \in P,\end{array}$ & $\begin{array}{c}\left(\left|V_{i}\right\rangle,\left|V_{j}\right\rangle,\left|W_{i}\right\rangle,\left|W_{k}\right\rangle,\left|U_{k}\right\rangle\right) \\
P=\{1,2,3\}\end{array}$ & $\begin{array}{c}\left(\left|V_{i}\right\rangle,\left|V_{j}\right\rangle,\left|W_{j}\right\rangle,\left|W_{k}\right\rangle,\left|U_{k}\right\rangle\right) \\
i \neq j \neq k\end{array}$ \\
\hline
\end{tabular}


Table 3. Classification of entangled states with six terms

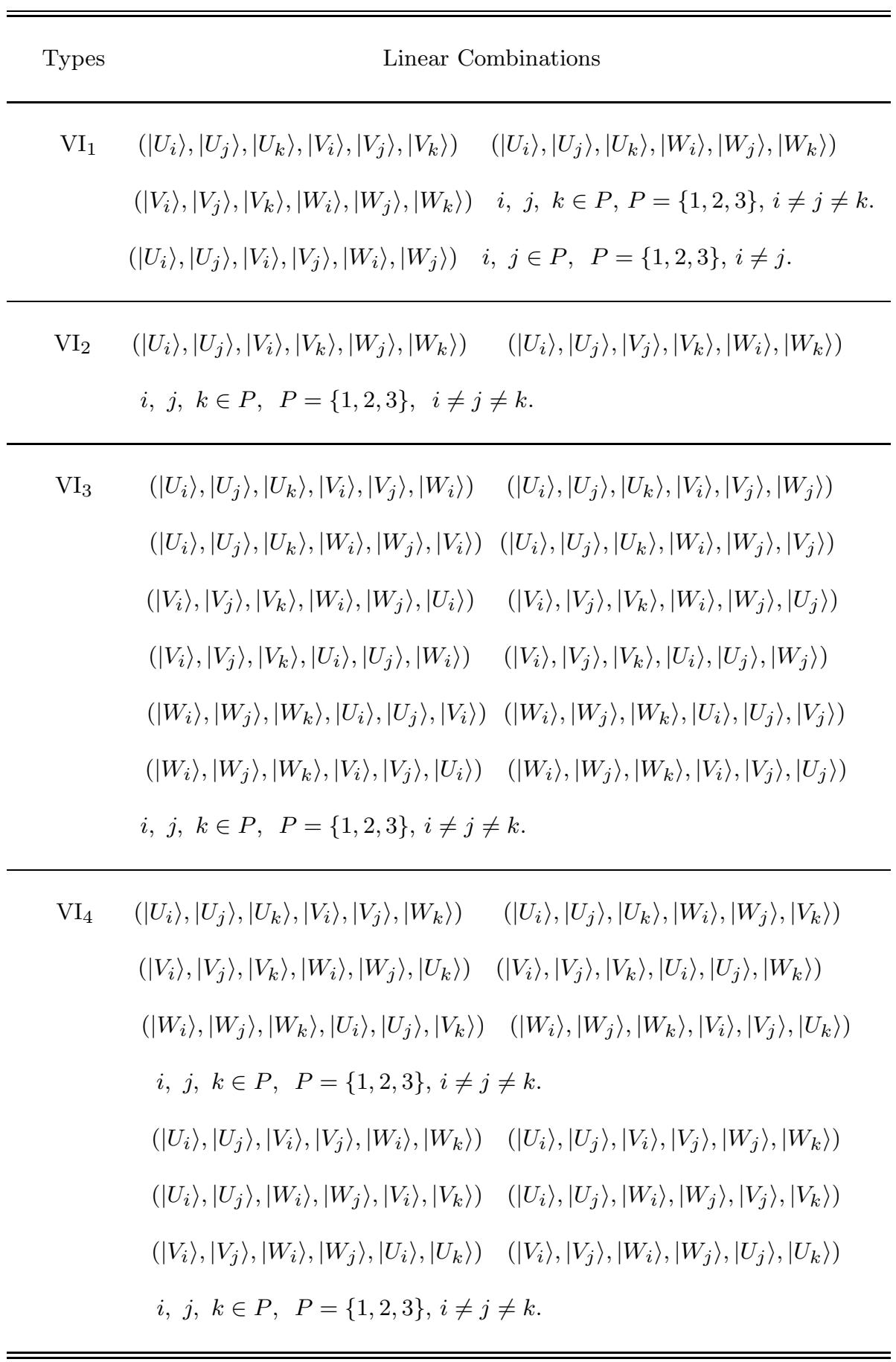


In fact, it can be proven that Type $\mathrm{VI}_{2}$ states are inequivalent to Type I and II states under SLOCC. To do so, we take

$$
|\Psi\rangle=\left(x_{1}|11\rangle+x_{2}|10\rangle+x_{3}|01\rangle+x_{4}|0-1\rangle+x_{5}|-10\rangle\right)+x_{6}|-1-1\rangle
$$

as an example with nonzero complex coefficients $\left(x_{1}, x_{2}, \cdots, x_{6}\right)$, where the five terms in the parentheses form the Type $V_{5}$ state, which is equivalent to a Type I state, while the remaining part is always equivalent to a bipartite product state under SLOCC. Since all coefficients are nonzero for the case considered, the state in (5) remains a linear combination of a Type I entangled state and a bipartite product state. It will degenerate into a Type I state only when $x_{6} \rightarrow 0$. Thus, it is proven that Type $\mathrm{VI}_{2}$ is inequivalent to a Type I state. Furthermore, (5) can also be written as

$$
\begin{gathered}
|\Psi\rangle=\left(\left(x_{1}|11\rangle+x_{6}|-1-1\rangle+x|00\rangle\right)\right. \\
\left.+\left(x_{2}|10\rangle+x_{3}|01\rangle+x_{4}|0-1\rangle+x_{5}|-10\rangle\right)-x|00\rangle\right),
\end{gathered}
$$

where $x$ is an arbitrary nonzero complex number. It is obvious that the terms in the first parentheses form a Type II state, while the terms in the second parentheses form a Type $\mathrm{V}_{4}$ state. Under SLOCC, a Type $\mathrm{V}_{4}$ state is always equivalent to a Type I state. Therefore, the state in (6) becomes a linear combination of a Type I and a Type II state if all coefficients $\left(x_{1}, x_{2}, \cdots, x_{6}\right)$ and $x$ are nonzero, which constitutes a proof that the $\mathrm{VI}_{2}$ is neither equivalent to a Type I state, nor equivalent to a Type II state under SLOCC. Hence the Type $\mathrm{VI}_{2}$ configuration is inequivalent to Type I and II states under SLOCC, which is called Type III. Similar analyses of Type $\mathrm{VI}_{3}$ and $\mathrm{VI}_{4}$ configurations was also carried out, from which we did not find any further new types of entangled states.

Let $C^{3}$ denote a Hilbert subspace of $S U(2)$ spanned by the single qutrit states. Then, $\left(C^{3}\right)^{\otimes N}$ is spanned by the N-particle product states. In this case, local unitary operations are elements among $U(1) \otimes(S U(2))^{\otimes N}$, where $U(1)$ provides an overall phase factor. According to the analysis shown in Ref. 16, there are $2 \times 3^{N}$ real parameters needed in a description of any vector in $\left(C^{3}\right)^{\otimes N}$. Any $S U(2)$ transformation needs three real parameters to describe it, and there is one more for $U(1)$. Therefore, the number of independent real parameters needed in a description of $\mathrm{LU}$ inequivalent type of states in the system is $2 \times 3^{N}-(3 N+1)$. For the bipartite case with $N=2$ considered in this paper, 11 real parameters are needed to describe an arbitrary state. Since 12 real numbers are needed in order to describe a state with six terms, our analysis on the classification of bipartite qutrit states with up to six terms provided is complete and sufficient. It follows that a similar analysis of states with more than 6 terms is not necessary.

In summary, by using the entanglement measure (1) with SLOCC transformations, a complete analysis for entangled bipartite qutrit pure states has been carried 
out. Three SLOCC inequivalent types of extremely entangled bipartite qutrit pure states have been identified by using constrained maximization. The extremal values of these three types of entanglement are $\eta=0.63093$ (Type I), $\eta=1$ (Type II), and $\eta=0.78969$ (Type III), respectively, corresponding to the following three forms under SLOCC:

$$
\begin{gathered}
|\mathrm{I}\rangle=\frac{1}{\sqrt{2}}(|11\rangle+|00\rangle), \quad \eta=0.63093 \\
|\mathrm{II}\rangle=\frac{1}{\sqrt{3}}(|11\rangle+|00\rangle+|-1-1\rangle), \quad \eta=1, \\
|\mathrm{III}\rangle=\frac{1}{\sqrt{6}}(|11\rangle+|-1-1\rangle+|10\rangle+|01\rangle+|0-1\rangle+|-10\rangle), \quad \eta=0.78969 .
\end{gathered}
$$

Our results show that the entanglement measure defined by (1) is also effective in classifying different genuinely entangled bipartite qutrit pure states. The most important evidence is that the number of basic ways of entanglement equals to the number of extremally entangled types, which is consistent to the conclusion shown in Ref. 13. Therefore, extremal entanglement is a necessary condition in finding different types of entanglement in multipartite pure state systems under SLOCC.

Support from the US National Science Foundation (0140300), the Southeastern Universitites Research Association, the Natural Science Foundation of China (10175031), the Natural Science Foundation of Liaoning Province (2001101053), the Education Department of Liaoning Province (202122024), and the LSU-LNNU joint research program (C164063) is acknowledged.

\section{References}

1. M. A. Nielsen and I. L. Chuang, Quantum Computation and Quantum Information (Cambridge Univ. Press, Cambridge, 2000).

2. D. Kaslikowski et al., Phys. Rev. Lett. 85, 4418 (2000).

3. D. Collins et al., Phys. Rev. Lett. 88, 040404 (2002).

4. H. Bechmann-Pasquinucci and A. Peres, Phys. Rev. Lett. 85, 3313 (2000).

5. T. Durt, N. J. Cerf, N. Gisin, and M. Zukowski, Phys. Rev. A 67, 012311 (2003).

6. M. Bourennane, A. Karlsson, and G. Björk, Phys. Rev. A 64, 012306 (2001).

7. N. J. Cerf, M. Bourennane, A. Karlsson, and N. Gisin, Phys. Rev. Lett. 88, 127902 (2002).

8. J. C. Howell, A. Lamas-Linares, and D. Bouwmeester, Phys. Rev. Lett. 85, 030401 (2002).

9. R. T. Thew, A. Acín, H. Zbinden and N. Gisin, Phys. Rev. Lett. 93, 010503 (2004).

10. A. Vaziri, G. Weihs, and A. Zeilinger, Phys. Rev. Lett. 89, 240401 (2002).

11. H. de Riedmatten, I. Marcikic, H. Zbinden and N. Gisin, Quant. Inf. and Comp. 2, 425 (2002).

12. F. Pan, D. Liu, G. Y. Lu, and J. P. Draayer, Int. J. Theor. Phys. 43, 1241 (2004).

13. F. Pan, D. Liu, G. Y. Lu, and J. P. Draayer, Phys. Lett. A336, 384 (2005). 
July 3, 2018 22:3 WSPC/INSTRUCTION FILE ijbpan4

10 F. Pan, G.-Y. Lu, and J. P. Draayer

14. W. Dür, G. Vidal, J. I. Cirac, Phys. Rev. A 62, 062314(2000).

15. C. H. Bennett, S. Popescu, D. Rohrlich, J. A. Smolin, A. V. Thapliyal, Phys. Rev. A 63 (2001) 012307.

16. N. Linden and S. Popescu, Fortsch. Phys. 46, 567 (1998). 